\title{
STUDIES OF CORONARY BLOOD FLOW BY MULTIPLE DYE DILUTION CURVES *
}

\author{
By THEOFILOS J. TSAGARIS, J. ALBRECHT KOEHLER, HIROSHI KUIDA, AND \\ HANS H. HECHT
}

\author{
(From the Department of Internal Medicine, University of Utah College of Medicine, \\ Salt Lake City, Utah)
}

(Submitted for publication May 28, 1962; accepted September 1, 1962)

The indicator-dilution technique has recently been applied to the study of the coronary circulation. One of the methods which have been proposed is the estimation of coronary blood flow from dilution curves sampled from the right ventricle after the injection of radio-iodinated human serum albumin into the left ventricle or into a catheter "wedged" in a branch of the pulmonary artery (1). Another is based on the claim that coronary blood flow can be quantitated from precordial radioactivity curves (2). These observations have been challenged on both experimental and theoretical grounds (3-6). The radioactive method has been re-examined by comparing intraarterial indicator dilution curves with those obtained by surface counting from the precordium and brain (7). In this study a prolonged disappearance slope obtained from the precordium when compared to brain and artery was found which was absent when the coronary circulation was seriously impaired. It was assumed that the slow passage of a radioactive indicator through the coronary bed might be one of the factors influencing the precordial dilution curve in spite of the small total amount circulating through this vascular compartment.

In the face of these uncertainties, it seemed of interest to examine the temporal relationship of an indicator dilution curve obtained from the coronary sinus with those from other intrathoracic cardiovascular sites.

In the present study, paired dye dilution curves were recorded simultaneously from multiple sites in the circulation, including the coronary sinus, after injection of the indicator either into a peripheral vein or into the chambers of the left side of the heart. The time relationships of such curves

* This study was supported in part by grants from the U. S. Public Health Service (HTS 5150) and the Utah Heart Association. were analyzed to determine if an indicator restricted to the coronary vascular bed could be identified in curves obtained from any site other than the coronary sinus. The results appear to show that with such an experimental design the blood flow through the coronary arterial system cannot be determined from dye dilution curves.

\section{METHOD}

Studies were performed in 16 dogs weighing between 13 and $29 \mathrm{~kg}$ (average $23.8 \mathrm{~kg}$ ). Animals were anesthetized with sodium pentobarbital ( $30 \mathrm{mg}$ per $\mathrm{kg}$ ), administered intravenously. Nine studies were carried out in animals with the chest intact. In these studies, three $50-\mathrm{cm}$ Cournand catheters were passed via the right external jugular vein and placed in the superior vena cava, coronary sinus, and pulmonary artery, respectively. A similar catheter was passed from the right femoral vein into the inferior vena cava so that the tip lay just above the diaphragm. A radio-opaque polyethylene catheter (Schick X-ray Co., no. 17,877-3) was passed via the right carotid artery into the left ventricle.

In six studies the chest was opened by splitting the sternum longitudinally. Respiration was maintained by a pressure-regulated, positive-pressure respirator. Special, fifteen-gauge, plastic cannulas, $6.25 \mathrm{~cm}$ in length and fitted with a needle stylus (Rochester Products Co., Rochester, Minn.), were inserted directly into the superior vena cava, inferior vena cava, and pulmonary artery. A short polyethylene catheter $(1.67 \mathrm{~cm} \mathrm{ID)}$ was inserted into the coronary sinus through the right atrial appendage and another into the left atrium through its appendage.

In the dogs with chest intact, 1.25 to $2.50 \mathrm{mg}$ indocyanine green dye was injected through the catheter into the left ventricle. The catheter was immediately flushed with saline after each injection. The dilution curves were recorded by densitometers (no. XC-100A, Waters Corp.). Paired curves were obtained as follows: coronary sinus (CS) and pulmonary artery (PA) (19 times), $\mathrm{CS}$ and inferior vena cava (IVC) (10 times), CS and superior vena cava (SVC) (2 times), PA and IVC (31 times), PA and SVC (12 times), and IVC and SVC (9 times). The same combinations of curves were obtained 40 times in the open-chest experiments after left 
atrial injection of similar amounts of indocyanine green dye.

A peripheral venous injection of $1.25 \mathrm{mg}$ of indocyanine green was made in three open- and in seven closed-chest experiments. Paired densitometer curves were obtained simultaneously from the PA and left ventricle (LV) or left atrium (LA). In two dogs (one open and one closed chest), LV (or aortic root) and CS densitometer curves were recorded simultaneously with PA curves obtained by means of a thermistor catheter (Victory Engineering Corp., Union, N. J.) after injection of cold indocyanine green dye $(0.63 \mathrm{mg}$ to $1.25 \mathrm{mg})$ into a peripheral vein. The experimental design is illustrated in Figure 1.

Blood was sampled through the densitometers from the appropriate catheters or cannulas by means of a constant rate sampling device consisting of paired syringes with motor driven plungers (8). The sampling rate varied between 0.3 and $0.5 \mathrm{cc}$ per second. The volume of each catheter was measured and corrections made for catheter dead space transit time. The dye dilution curves were recorded either at 5 or $25 \mathrm{~mm}$ per second paper speed on a Visicorder (Minneapolis-Honeywell Co., Denver, Colo.). The appearance time of the curves could be determined accurately to within 0.25 seconds at the faster speed and to within 0.5 seconds at the slow speed. The dynamic response of the $50-\mathrm{cm}$ catheter-densitometergalvonometer system using a sampling rate of $0.4 \mathrm{ml}$ per second was such that it responded to a step change in dye concentration by achieving 63 per cent of the final deflection (time constant) in 0.8 seconds. With the short cannulas used in the open-chest preparations and the same sampling rate, the time constant was 0.3 seconds. The thermistor probes responded to a sudden temperature change (still water) with a time constant of 0.6 seconds. These responses are considered more than adequate to follow, with fidelity, even the most rapid changes in concentration of dye that occurred in the various preparations.

In two of nine closed-chest experiments coronary sinus catheterization was unsuccessful. In two others, even though the catheter was properly placed in the coronary sinus, acceptable dye dilution curves could not be obtained because of erratic sampling rate. Correct placement of the CS catheter was determined by the characteristic position of the catheter on fluoroscopy (9) and by the marked desaturation of blood (by gas analysis) sampled from it.

\section{RESULTS}

The results of this study are based on the analysis of one hundred and seventy pairs of dye curves.

A. Appearance time. The analysis of appearance time $\left(\mathrm{t}_{\mathrm{a}}\right)$ of indicator in the CS, PA, SVC, and IVC after left atrial or left ventricular injection is shown in Table I. Dye consistently appeared earliest in the CS and last in the SVC

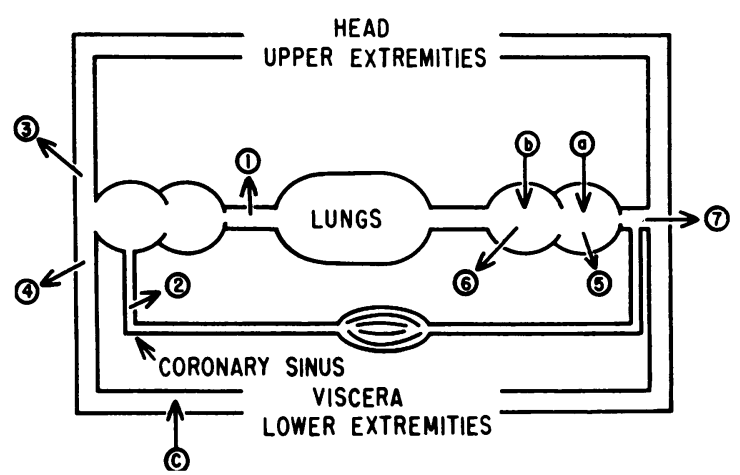

Fig. 1. Schematic diagram of the circulation WITH THE MULTIPLE SAMPLING SITES IDENTIFIED BY NUMBERS (1-7) AND INJECTION SITES BY LETTERS (A-C). Injection was made into left ventricle (a), left atrium (b), and peripheral vein (c). Samples were obtained from pulmonary artery (1), coronary sinus (2), superior vena cava (3), inferior vena cava (4), left ventricle (5), left atrium (6), and ascending aorta (7). (See text.)

(Figure 2). Shortly after its appearance in the coronary sinus, dye appeared virtually simultaneously in the PA and IVC (Figure 2). In sereral dogs (both open- and closed-chest preparations), $t_{a}$ of the SVC curve was markedly delayed despite an adequate sampling rate. Most of the IVC curves had a smooth buildup and disappearance slope, but a few had a multiple spike contour (Figure 3). This peculiar pattern, first consiclered an artifact, could be reproduced repeatedly. during a particular study in the same animal even though densitometers were interchanged and other possible technical errors were eliminated.

B. Contour of PA curves. The contour of ninety-two PA curves after left atrial or left ventricular injection was analyzed in an attempt to separate dye carried by coronary blood flow from that of all "noncoronary" circuits. In none of the PA curves analyzed was there a "hump" on the ascending limb which could have suggested an early contribution to the PA curve of dye coming from a vascular bed, presumably the coronary circulation, with a transit time significantly shorter than the average. Since the presence of a catheter in the coronary sinus conceivably could have impeded normal coronary blood flow, PA curves were obtained with and without a catheter in the CS. Pulmonary artery curves obtained under these circumstances were similar.

C. Temporal relationship between $P A, L V$, and $C S$ curves. The temporal relationship between dye 


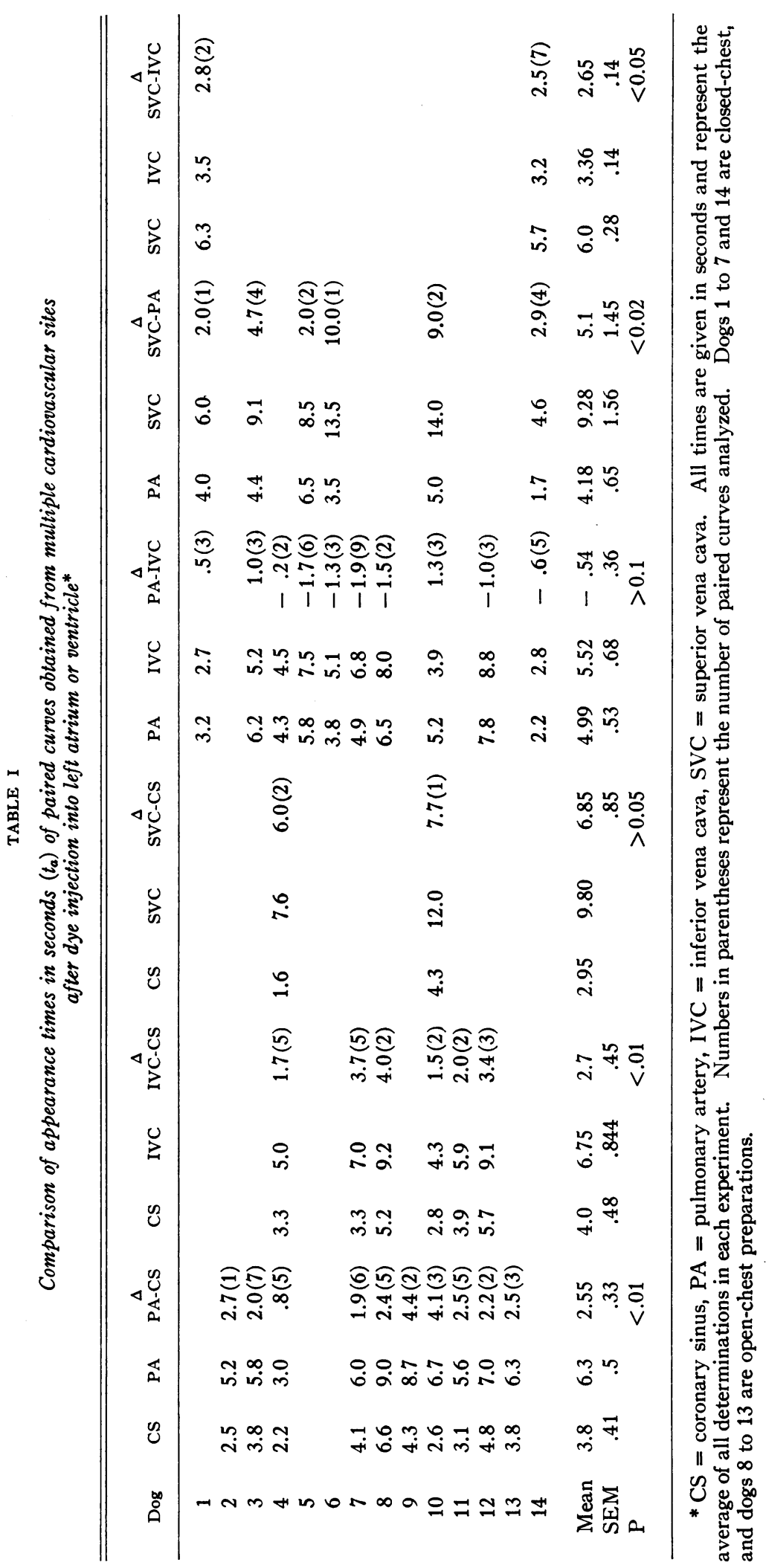


LV Inj.

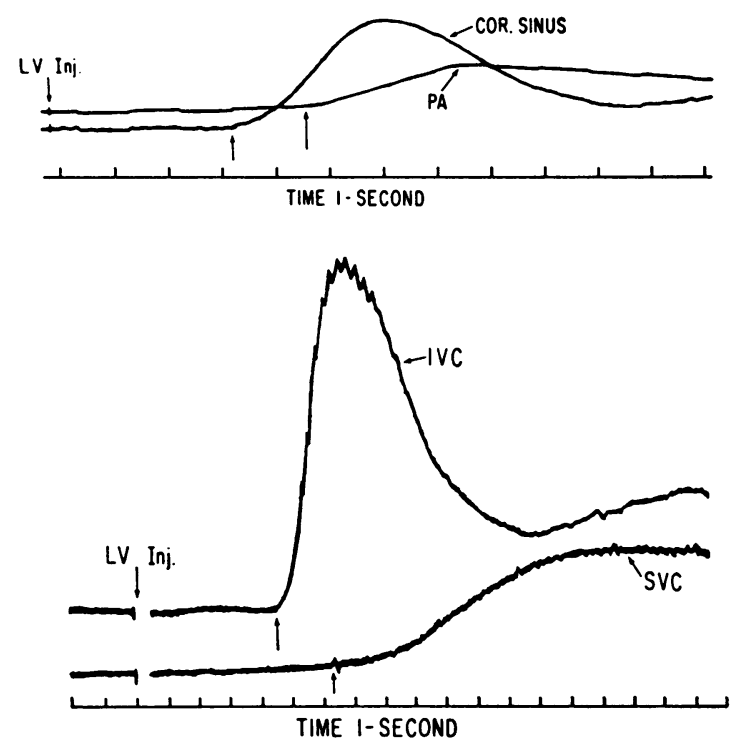

LV Inj.
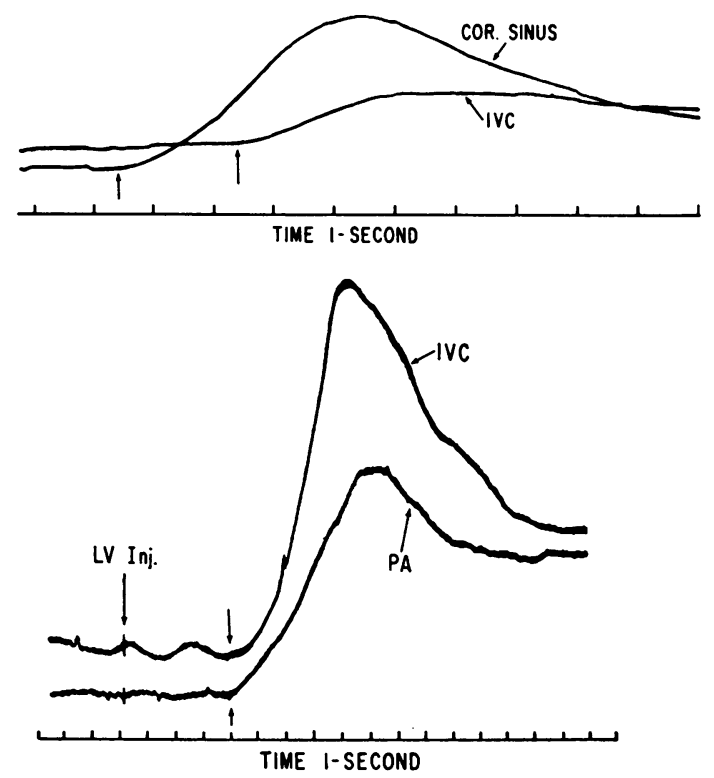

Fig. 2. Four different combinations of DILUtion CURVES obtained in Simultaneous pairs from indicated SITES AFTER INJECTION OF DYE INTO THE LEFT VENTRICLE (LV). The relationship of appearance time of indicator (indicated by arrows) of each pair is demonstrated.

curves obtained simultaneously from the PA, LV, and CS was carefully evaluated after dye injection into a peripheral vein. Thirty pairs of densitometer curves were obtained simultaneously from the PA and LV. Seventeen sets of three simultaneous curves were obtained from the PA (thermistor), CS (densitometer), and LV or aortic root (densitometer). The progressive increase in the time base of each curve as the indicator proceeds away from the injection site results in an overlap among the ascending limb of the CS curve, the primary LV curve, and the recirculation component of the PA curve. In addition, the descending limb of the coronary sinus curve coincides with the recirculation of the LV curve (Figure 4).

The total amount of indicator emerging from the CS is but a small fraction ( 5 to 10 per cent) of that leaving the PA or LV. In order to demonstrate this quantitative relationship, Figure 5 has been constructed with the same time base for each curve as in Figure 4, but it now transforms the concentration of dye as measured by the optical density of blood to total amount of dye (in milligrams). The upper dotted curve of Figure 5A represents the total amount of indicator present in the heart or great vessels at any given moment, the lower dotted curve, the total amount in the absence of dye in the coronary bed. When indicator is present in the coronary vascular bed, it represents only a small fraction of the total amount of indicator in the cardiac chambers or in the proximal segment of the great vessels.

\section{DISCUSSION}

The PA curve which is recorded after a left atrial or left ventricular injection represents the net result of dye having traversed all possible systemic vascular beds. The transit time of each particle depends on the length of the particular vascular bed traversed by the particle, the mean

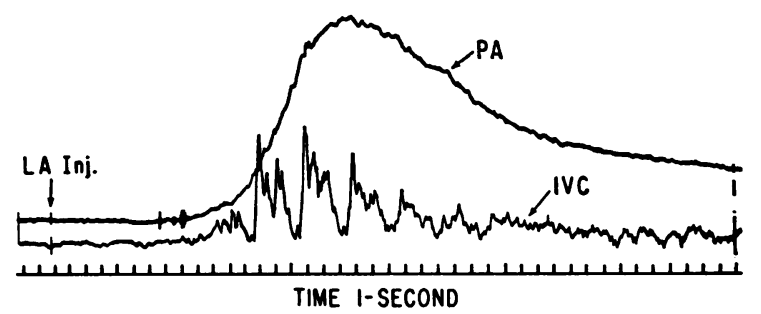

Fig. 3. Dye curves obtained simultaneously from THE PULMONARY ARTERY (PA) AND INFERIOR VENA CAVA (IVC). The latter shows a "multiple spike" contour, observed consistently in some animals. (See text.) 


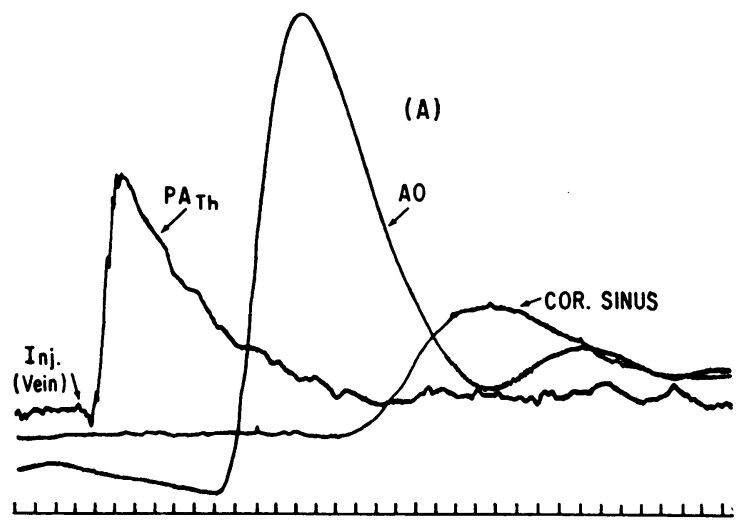

TIME I-SECOND

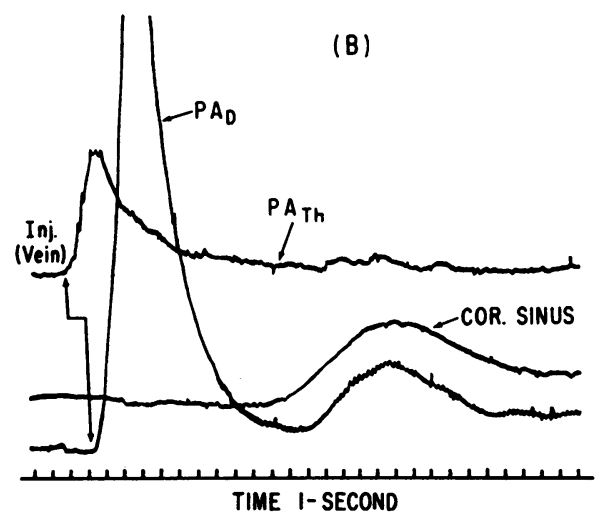

Fig. 4. Temporal Relationship of CURVEs obtained FROM THE PULMONARY ARTERY WITH A THERMISTOR $\left(\right.$ PA $_{\text {TH }}$ ) AND FROM PULMONARY ARTERY (PAD), AORTIC ROOT (AO), AND CORONARY SINUS (COR. SINUS) WITH DENSITOMETERS AFTER THE INJECTION OF COLD DYE INTO A PERIPHERAL VEIN. The appearance time of dye in the $\mathrm{PA}$ indicated by the broken arrow in (B) is delayed in the densitometer curve $\left(\mathrm{PA}_{\mathrm{D}}\right)$ compared with the thermistor curve $\left(\mathrm{PA}_{\mathrm{th}}\right)$ owing to the sampling dead space of the former system. Note: The coronary sinus curve is superimposed on the primary aortic curve and on the recirculation curves of both aorta and pulmonary artery.

cross-sectional area of the vascular bed, and the flow rate through it. The fraction of the injected indicator returning from each bed is assumed to be directly proportional to the fraction of cardiac output perfusing each bed. Because its transit time is shortest, at least as judged by $t_{a}$, it might be anticipated that dye passing through the coronary circulation could be identified as an early component of the PA curve. This, in essence, was Henly's assumption (although RV was the sampling site). In our experiments, however, the difference between the transit times of the coronary vascular bed and one or more beds which drain into the inferior vena cava is too small to permit a clear separation of coronary flow from other drainage areas (Table I). In addition, the total amount of indicator (in milligrams) emerging from the coronary sinus is only a small fraction of that returning from the inferior vena cava (10), which in our experiments showed the earliest appearance time of dye passing through noncoronary vascular regions. The combination of these two factors is probably sufficient to explain our inability to detect in the PA curve indicator which traversed only the coronary circulation. This situation obtains even when conditions are optimal for isolating "coronary" dye, namely, left heart injection of indicator. The more distant from the aortic root injection is made, the more dispersed the primary curve entering the coronary circulation would be.

Occasionally dye appearance from the IVC was
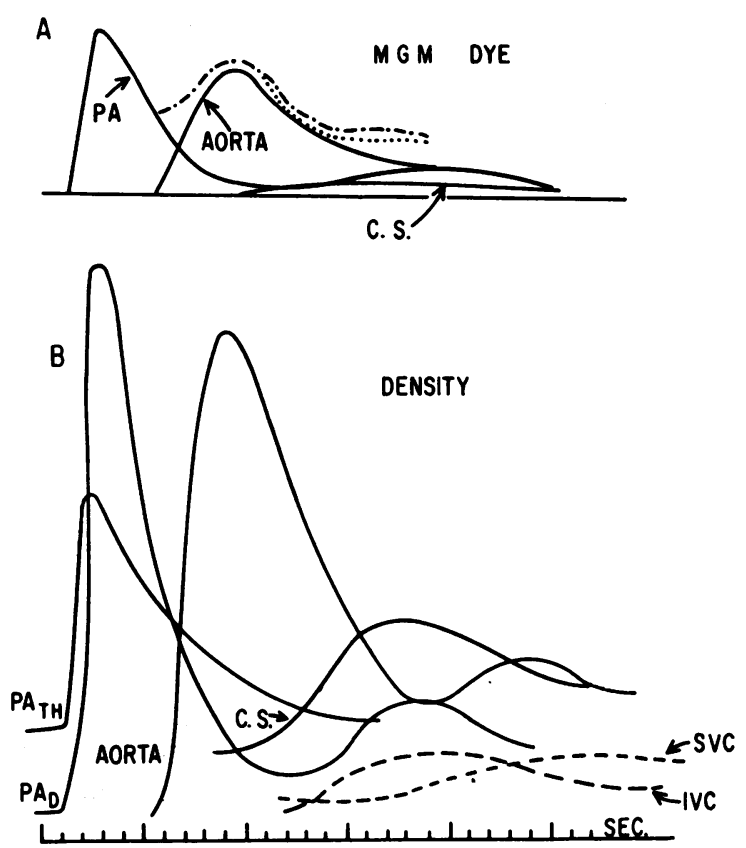

Fig. 5. A. Hypothetical curves of total QUANTity RATHER THAN CONCENTRATION OF INDICATOR TRAVERSING vascular SITES AS InDicated. The upper dotted line represents total amount of dye in heart and great vessels including those in the coronary vascular bed, the lower dotted line, total amount of dye excluding the coronary circulation. B. COMPOSITE REDRAWN FROM CURVES SHOWN IN Figure 4 to SHOW RELATIONShIP OF PRIMARY AND RECIRCULATION CURVES OBTAINED FROM THE PULMONARY ARTERY (PA), AORTIC ROOT (AO), CORONARY SINUS (CS), INFERIOR VENA CAVA (IVC), AND SUPERIOR VENA CAVA (SVC) AFTER PERIPHERAL VENOUS INJECTION OF COLD DYE. IVC and SVC curves have been added using appearance time relationships shown in Table $\mathrm{I}$. 
difficult to determine because of a peculiar "multiple spike" contour of IVC curves observed consistently in the same three animals (Figure 3 ). Such spikes apparently do indicate true return of dye, since technical errors seem to have been excluded. They occurred even though artificial respiration was temporarily discontinued. They may represent indicator returning from the various beds drained by the inferior vena cava in streamlines of varying concentrations of dye. Placement of the catheter tip in a stream draining a bed with either a long or short transit time could result either in a delayed or in an early appearance of indicator, and the absence of a smooth curve might therefore be a consequence of incomplete mixing.

The relationships of dye curves obtained from the PA, LV, and CS after peripheral venous injection of indicator is determined by the progressive dispersion of the original bolus of indicator and by the path traversed. Analysis of the LV curve after venous injection reveals that the input function of dye concentration to the coronary arteries is distributed over several seconds. The output curve from the coronary vascular bed is then further "smeared." Indicator traversing the entire coronary circulation would of necessity be distributed over a time base encompassing the input and output curves and would include no more than 5 to 10 per cent of the total quantity injected. This small amount of dye distributed over a long transit period is submerged in different phases of the primary and recirculation curves in the left ventricle and pulmonary artery.

Isolation of indicator restricted to the coronary circulation by precordial counting of radioactivity requires that the indicator traversing this bed is of sufficient magnitude to be detected by the sensing device and its appearance is temporally separable from indicator present in the heart or great vessels. In the present experiments, the small fraction of the injected dye reaching the coronary bed combined with its dispersion over a broad time base seems to preclude the possibility of separating indicator restricted solely to the coronary circulation.

\section{SUMMARY}

1. Isolation of indicator restricted to the coronary vascular bed has been attempted after peripheral venous and left atrial or left ventricular injection.
2. After left atrial or left ventricular injection, the inability to identify indicator emerging from the coronary bed in pulmonary artery curves is ascribed to $a$ ) the small fraction of total injected indicator traversing this bed and $b$ ) the small difference in $t_{a}$ of indicator in the coronary sinus and the noncoronary vascular beds.

3. The temporal relationship of dye curves in multiple cardiac sites has been analyzed in terms of approximate quantity of indicator rather than concentration traversing each site. Results suggest that methods which attempt to isolate indicator restricted to the coronary vascular bed by means of precordial monitoring of radioactivity would be compromised by the simultaneous presence of large quantities of indicator in the heart and noncoronary vessels in the immediate vicinity.

\section{REFERENCES}

1. Henly, W. S., Creech, O., Jr., Couves, C. M., Morgan, M. C., Chapman, Don W., and Allen, H. C., Jr. Determination of myocardial blood flow utilizing iodinated $\left(\mathrm{I}^{131}\right)$ human serum albumin. Surg. Forum 1956, 7, 306.

2. Sevelius, G., and Johnson, P. C. Myocardial blood flow determined by surface counting and ratio formula. J. Lab. clin. Med. 1959, 54. 669.

3. Marchioro, T., Feldman, A. J., Owens, C. J., and Swan, H. Measurement of myocardial blood flow : indicator dilution technique. Circulat. Res. 1961, 9, 541.

4. Forte, I. E., Schmitthenner, J. E., and Neal, H. S. Measurement of coronary blood flow using radioactive iodine compared with nitrous oxide. Circulat. Res. 1961, 9, 547.

5. Marshall, R. J. Factors modifying the contour of indicator-dilution curves. Circulat. Res. 1962, 10, 123.

6. Conn, H. L., Jr. Use of external counting techniques in studies of the circulation. Circulat. Res. 1962, 10, 505.

7. Mena, I., Kattus, A. A., Greenfield, M. A., and Bennett, L. R. Effect of coronary blood flow on radioisotope dilution curves measured by precordial scintillation detection. Circulat. Res. 1961, 9, 911.

8. Lange, R. L., and Hecht, H. H. Quantitation of valvular regurgitation from multiple indicator dilution curves. Circulation 1958, 18, 623.

9. Goodale, W. T., Martin, L., Eckenhoff, J. E., Hafkenschiel, J. H., and Banfield, W. G. Coronary sinus catheterization for studying coronary blood flow and myocardial metabolism. Amer. J. Physiol. $1948,152,340$.

10. Fox, I. J., and Wood, E. H. Indicator-dilution techniques in study of normal and abnormal circulation in Medical Physics, O. Glasser, Ed. Chicago, Year Book Publishers, Inc., 1960, vol. 3, p. 163. 